\title{
Resistant mechanisms and molecular epidemiology of imipenem-resistant Acinetobacter baumannii
}

\author{
SHU-ZHEN XIAO ${ }^{1 *}$, HAI-QING CHU ${ }^{2}$, LI-ZHONG HAN ${ }^{1 *}$, \\ ZHE-MIN ZHANG $^{2 *}$, BING $\mathrm{LI}^{2}$, LAN ZHAO ${ }^{2}$ and LIYUN XU ${ }^{2}$ \\ ${ }^{1}$ Department of Clinical Microbiology, Ruijin Hospital, Shanghai Jiaotong University School \\ of Medicine, Shanghai 200025; ${ }^{2}$ Department of Respiratory Medicine, Shanghai Pulmonary \\ Hospital, Tongji University School of Medicine, Shanghai 200433, P.R. China
}

Received January 5, 2016; Accepted July 22, 2016

DOI: $10.3892 / \mathrm{mmr} .2016 .5538$

\begin{abstract}
The aim of the study was to investigate the resistant mechanisms and homology of imipenem-resistant Acinetobacter baumannii (A. baumannii). A total of 46 non-duplicate imipenem-resistant $A$. baumannii clinical isolates were collected from three tertiary hospitals between July,2011 and June, 2012.The minimal inhibitory concentrations (MICs) of antimicrobial agents were determined using the agar dilution method. Phenylalanine-arginine $\beta$-naphthylamide was used to detect the presence of the efflux pump-mediated resistant mechanism. Polymerase chain reaction was employed to amplify genes associated with drug resistance, including $\beta$-lactamase genes, efflux pump genes and outer membrane protein gene $\operatorname{CarO}$. A few amplicons were randomly selected and sequenced. Multilocus sequence analysis (MLST) was employed in typing A. baumanni. A. baumannii was resistant to imipenem, simultaneously showing resistance to several other antimicrobials. In addition, 13 A. baumannii were found to mediate drug resistance through operation of the efflux pump. Of the various drug resistance genes tested, $b l a_{\text {OXA-51 }}$ was present in 46 isolates, $b l a_{\text {OXA-23 }}$ gene was present in 44 isolates and $b l a_{\mathrm{NDM}}$ gene was found in only one strain. Other drug resistant-associated genes, including $b l a_{\mathrm{KPC}}$, $b l a_{\mathrm{IMP}}, b l a_{\mathrm{OXA}-24}, b l a_{\mathrm{OXA}-58}, b l a_{\mathrm{SHV}}, b l a_{\mathrm{GIM}}$ and $b l a_{\mathrm{VIM}}$ were not detected. Mutation of adeS and outer membrane protein gene $\mathrm{CarO}$ were found in a few of the imipenem-resistant isolates. The MLST analysis revealed that all 46 clinical isolates were clustered into 11 genotypes and the most frequent genotype
\end{abstract}

Correspondence to: Dr Hai-Qing Chu, Department of Respiratory Medicine, Shanghai Pulmonary Hospital, Tongji University School of Medicine, 507 ZhengMin Road, Shanghai 200433, P.R. China E-mail: nqy320@163.com

${ }^{*}$ Contributed equally

Key words: Acinetobacter baumannii, imipenem, efflux pump, minimal inhibitory concentration, multilocus sequence analysis, molecular epidemiology was ST208. In conclusion, $\beta$-lactamase genes, genes involved in efflux pump and mutation of outer membrane protein encoding gene may be important in mediating imipenem resistance in A. baumannii. Of the 11 different genotypes, ST11 was shared by the majority of $A$. baumannii, which may be due to horizontal transfer of patients from hospitals.

\section{Introduction}

Acinetobacter baumannii (A. baumannii) has emerged as a major pathogen of nosocomial infections and is associated with high rates of morbidity and mortality in recent years $(1,2)$. A nationwide surveillance program, including hospitals from 14 geographically different regions in China revealed that the ratio of A. baumannii is on the increase annually (3).

Carbapenem has good antibacterial activity against A. baumannii and was the first choice in treatment of infection caused by A. baumannii in the past years (4). However, the emergence of resistance to carbapenem was reported in 1991 (5), followed by similar reports from different parts of the world (6,7). In China, 57 and $61 \%$ of Acinetobacter spp. (A. baumannii accounted for 89.6\%) showed resistance to imipenem and meropenem, respectively (3). International studies in China as well as in other parts of the world focused only on evaluating the resistance of $A$. baumannii to various antimicrobials (8-10). However, to the best of our knowledge, few studies have investigated the molecular mechanism underlying drug resistance. Additionally, no data are available on the epidemiological characteristics of imipenem-resistant A. baumannii in Shanghai.

Thus, A. baumannii clinical isolates were collected from three tertiary hospitals in Shanghai and their drug resistance pattern to a spectrum of antimicrobials, molecular mechanisms (including carbapenemase, efflux pumps and membrane proteins) behind their resistance and multilocus sequence analysis (MLST) were analyzed to assess their molecular epidemiology.

\section{Materials and methods}

Bacterial strains. During the period July, 2011 to June, 2012, 46 non-duplicate imipenem-resistant $A$. baumannii strains 
Table I. Gene-specific primers used in this study.

\begin{tabular}{|c|c|}
\hline Genes & Primer sequences \\
\hline \multirow[t]{2}{*}{ recA } & F: CCTGAATCTTCTGGTAAAAC \\
\hline & R: GTTTCTGGGCTGCCAAACATTAC \\
\hline \multirow[t]{2}{*}{ ITS } & F: CATTATCACGGTAATTAGTG \\
\hline & R: AGAGCACTGTGCACTTAAG \\
\hline \multirow{2}{*}{$b l a_{\mathrm{KPC}}$} & F: TTACTGCCCGTTGACGCCCAATCC \\
\hline & R: TCGCTAAACTCGAACAGG \\
\hline \multirow{2}{*}{$b l a_{\mathrm{IMP}}$} & F: AACCAGTTTTGCCTTACCAT \\
\hline & R: CTACCGCAGCAGAGTCTTTG \\
\hline \multirow{2}{*}{$b l a_{\mathrm{NDM}}$} & F: CCGCCCAGATCCTCAACT \\
\hline & R: ATCAGGCAGCCACCAAAA \\
\hline \multirow{2}{*}{$b l a_{\text {OXA-51 }}$} & F: TAATGCTTTGATCGGCCTTG \\
\hline & R: TGGATTGCACTTCATCTTGG \\
\hline \multirow{2}{*}{$b l a_{\text {OXA-23 }}$} & F: GATCGGATTGGAGAACCAGA \\
\hline & R: ATTTCTGACCCATTTCCAT \\
\hline \multirow{2}{*}{$b l a_{\text {OXA-24 }}$} & F: GGTTAGTTGGCCCCCTTAAA \\
\hline & R: AGTTGAGCGAAAAGGGGATT \\
\hline \multirow[t]{2}{*}{$b l a_{\text {OXA-58 }}$} & F: AAGTATTGGGGCTTGTGCTG \\
\hline & R: CCCCTCTGCGCTCTACATAC \\
\hline \multirow[t]{2}{*}{$b l a_{\mathrm{SHV}}$} & F: GGTTATGCGTTATATTCGCC \\
\hline & R: TTAGCGTTGCCAGTGCTC \\
\hline \multirow[t]{2}{*}{$b l a_{\mathrm{GIM}}$} & F: AGAACCTTGACCGAACGCAG \\
\hline & R: ACTCATGACTCCTCACGAGG \\
\hline \multirow[t]{2}{*}{$b l a_{\mathrm{VIM}}$} & F: TCCACGCACTTTCATGACGA \\
\hline & R: AGACGTGCGTGACAACTCAT \\
\hline \multirow[t]{2}{*}{ adeA } & F: GAAATCCGTCCGCAAGTC \\
\hline & R: ACACGCACATACATACCC \\
\hline \multirow[t]{2}{*}{$a d e B$} & F: AAAGACTTCAAAGAGCGG \\
\hline & R: TCACGCATTGCTTCACCC \\
\hline \multirow[t]{2}{*}{ adeC } & F: ATTTCAGGTCGTAGCATT \\
\hline & R: CTTGATAAGTAGAGTAGGGATT \\
\hline \multirow[t]{2}{*}{ ades } & F: ACTGTTATCTTCTGTGGCTGTA \\
\hline & R: GTGGACGTTAGGTCAAGTTCTG \\
\hline \multirow[t]{2}{*}{ adeR } & F: AAACGGTTGGGAAGTATTA \\
\hline & R: ATGGCTATCTACGGTTCG \\
\hline \multirow[t]{2}{*}{$\mathrm{CarO}$} & F: AAGGAGAAAACGATGA \\
\hline & R: TTATTACGTGGTTATGG \\
\hline \multirow[t]{2}{*}{ gltA } & F: AATTTACAGTGGCACATTAGGTCCC \\
\hline & R:GCAGAGATACCAGCAGAGATACACG \\
\hline \multirow[t]{2}{*}{ gyrB } & F: TGAAGGCGGCTTATCTGAGT \\
\hline & R: GCTGGGTCTTTTTCCTGACA \\
\hline \multirow[t]{2}{*}{$g d h B$} & F: ACCACATGCTTTGTTATG \\
\hline & R: GTTGGCGTATGTTGTGC \\
\hline \multirow[t]{2}{*}{ recA } & F: CCTGAATCTTCYGGTAAAAC \\
\hline & R: GTTTCTGGGCTGCCAAACATTAC \\
\hline \multirow[t]{2}{*}{ cpn60 } & F: GGTGCTCAACTTGTTCGTGA \\
\hline & R: CACCGAAACCAGGAGCTTTA \\
\hline Gpi & F: GAAATTTCCGGAGCTCACAA \\
\hline & R: TCAGGAGCAATACCCCACTC \\
\hline rpoD & F: ACCCGTGAAGGTGAAATCAG \\
\hline & R: TTCAGCTGGAGCTTTAGCAAT \\
\hline
\end{tabular}

were collected from three tertiary hospitals located in Shanghai, China.

Reconfirmation of strains. The collected strains were subjected to gram staining, biochemical tests, and recA gene and $16 \mathrm{~S}-23 \mathrm{~S}$ rRNA gene intergenic spacer region to reconfirm them as A. baumannii (7).

Antimicrobial susceptibility and efflux phenotype tests. The collected A. baumannii isolates were subjected to an antimicrobial susceptibility test against imipenem, meropenem, amikacin, piperacillin, ceftazidime, cefotaxime, minocycline, ciprofloxacin, ampicillin/sulbactam, sulbactam, cefoperazone/sulbactam, piperacillin/tazobactam, colistin, tigecycline and trimethoprim/sulfamethoxazole using agar dilution method. Escherichia coli strain ATCC25922 and Pseudomonas aeruginosa (P. aeruginosa) strain ATCC27853 were used as reference strains.

Strains in which efflux pump operation was detected by agar dilution method where imipenem- and meropenem-resistant isolates were cultured in Mueller-Hinton agar contained the efflux pump inhibitor phenylalanine-arginine $\beta$-naphthylamide $(\mathrm{PA} \beta \mathrm{N})$ at a final concentration of $20 \mathrm{mg} / \mathrm{l}(11,12)$. A $\geq 4$-fold reduction of imipenem or meropenem minimal inhibitory concentrations (MICs) in the presence of PA $\beta \mathrm{N}$ possessed an operating drug efflux pump.

Analysis of genes responsible for drug resistance, drug efflux and outer membrane protein. Polymerase chain reaction (PCR) was performed for the genes, $b l a_{\mathrm{KPC}}, b l a_{\mathrm{IMP}}, b l a_{\mathrm{NDM}}, b l a_{\mathrm{OXA}-51}$, bla $a_{\mathrm{OXA}-23}, b l a_{\mathrm{OXA}-24}, b l a_{\mathrm{OXA}-58}, b_{\mathrm{SHV}}$, bla $_{\mathrm{GIM}}$ and bla $_{\mathrm{VIM}}$, CarO, ade $A$, adeB, ade $C$, adeS and adeR. Thus, obtained amplicons were subjected to sequencing analysis.

A fresh and pure bacterial colony was suspended in distilled water and boiled at $100^{\circ} \mathrm{C}$ for $15 \mathrm{~min}$. After centrifugation at $8,000 \mathrm{x} \mathrm{g}$ for $15 \mathrm{~min}, 1 \mu \mathrm{l}$ of the supernatant was used for PCR analysis with the primers (Table I). PCR was performed in a total volume of $50 \mu \mathrm{l}$ containing $0.25 \mu \mathrm{l}$ Taq DNA polymerase (Takara Bio, Inc., Tokyo, Japan), $5 \mu 1$ 10X PCR buffer ( $\mathrm{Mg}^{2+}$ Plus), $4 \mu \mathrm{l} \mathrm{dNTP}$ mixture $(2.5 \mathrm{mM}$ each), $2.5 \mu \mathrm{l}$ DNA template, $1 \mu \mathrm{l}$ of each primer $(20 \mu \mathrm{M})$, and

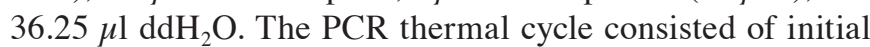
denaturation at $94^{\circ} \mathrm{C}$ for $5 \mathrm{~min}$, followed by 30 cycles of $94^{\circ} \mathrm{C}$ for $30 \mathrm{sec}$, annealing $55^{\circ} \mathrm{C}$ for $1 \mathrm{~min}$ and $72^{\circ} \mathrm{C}$ for $1 \mathrm{~min}$ and a final extension at $72^{\circ} \mathrm{C}$ for $7 \mathrm{~min}$. The PCR products were electrophoresed in $1 \%$ agarose gel and visualized under ultraviolet light, and subsequently sequenced (Sangon Biotech Co., Ltd., Shanghai, China).

MLST. Seven housekeeping genes including homologous recombination factor $(\mathrm{rec} A)$, citrate synthase ( $g l t A)$, DNA gyrase subunit $($ gyrB $)$, glucose-6-phosphate isomerase isomerase (gpi), glucose dehydrogenase B $(g d h B), 60-\mathrm{kDa}$ chaperonin (cpn60), and RNA polymerase 70 factor $(r p o D)$ were amplified in PCR using relevant primers (Table I) and appropriate thermal conditions. The amplicons were sequenced and the sequences were submitted to the MLST database (http://pubmlst.org.net) to compare them with sequences submitted from other parts of the world. Each strain was then characterized by a pattern of numbers defining its allelic profile. 
A
Query 61
aTTAGTCACGGCGACCTCTCTGCTAGAGCTTACGATAATAGAATTCACTCCGCCGAAATG
Sbjet 2041963
|||||||||||||||||||||||||||||||||||||||||||||||||||||||||| $\mid$
$\begin{array}{ll}\text { Query } & 121 \\ \text { Sbjct } & 2042023\end{array}$
TCGGAGCTTTTATATAATTTTAATGATATGGCTChHAGCTAGAGGTTCTGTTAHAHAT |l|||||||||||||||||||||||||||||||||||||||||||| |||||||
Sbjet 2042023
Query 181

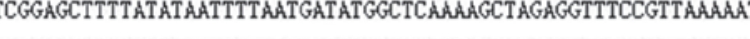
Sbjet 2042083
GCGCAGGTTTGGATGCAGCCATCGCACATGAGTTAAGALGCCTATAACGATATTACAA 240 |||||||||||||||||||||||||||||||||||||||||||||||||||||||| $\mid$
Query 241
GGTCGTTTACAAGGCATCATCGACGGTGTTTTTAHACCTGATGAAGTCCTATTTAAAAGC 300
Sbjet 2042143 GGTCGTTTACAGGGATTATTGATGCGTTTTAhACTTGATGAGTTCTATTTAhAGT
Query 301
Sbjet 2042203
CTTTPAHACAGTTGAHGTTTTATCTCACTTAGTCGAGGACTTACGGACTTTAGCTTA 360

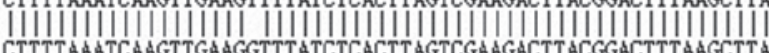
Query 361
GTAGAGACCAGCAACTCCGGTTAHATTATGAATTGTTTGACTTTAAGGCGTAGTTGAA 420
Sbjet 2042263

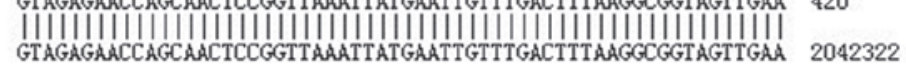
Query 421
AAAGTTCTTAAAGCATTTGAAGATCGITTGGATGAHGCTAAGCTAGTACCAGAACTTGAC

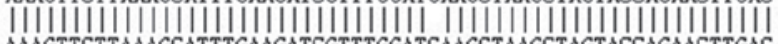
Sbjet 2042323
AhAGTTCTTHALGATTTGAGATCGTTTGGATChHGCTHAGCTAGTACCAGALTTGAC 2042382

B

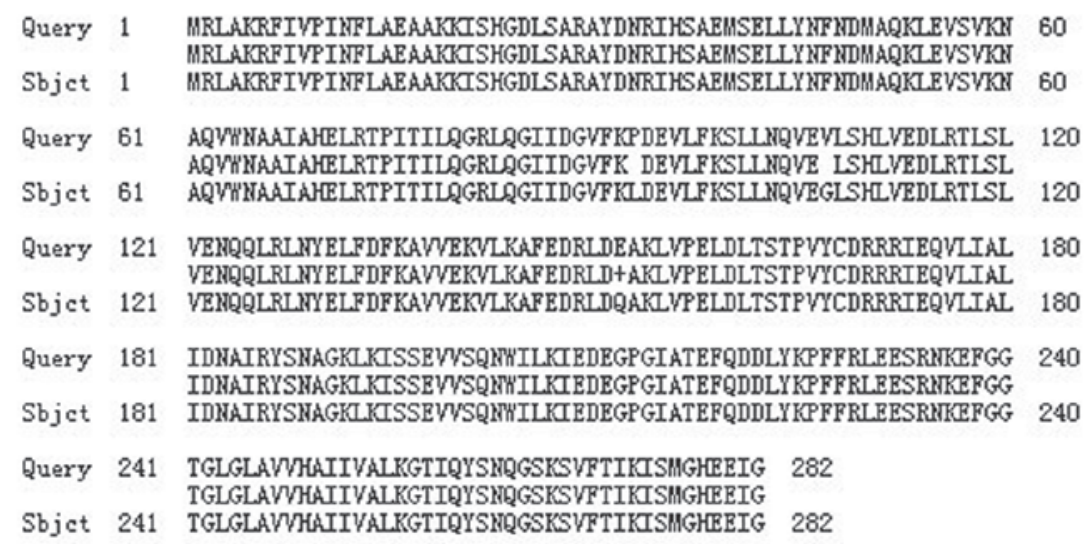

Figure 1. (A) Comparison of nucleotide sequence of $a d e S$ gene between resistant strain and reference strain ATCC17978. (B) Comparison of amino acid sequence of adeS gene between resistant strain and reference strain ATCC17978.

\section{Results}

Antimicrobial susceptibility. A. baumannii resistant to imipenem simultaneously showed resistance to several other common antimicrobials. The resistance rate was $>80 \%$ for all the antimicrobials except minocycline and colistin. Antibiotic susceptibility of the 46 clinical isolates is shown in Table II. Thirteen imipenem-resistant A. baumannii isolates were positive for efflux pump.

Detection of genes involved in drug resistance, drug efflux and outer membrane protein. Of the various drug resistance genes tested, $b l a_{\mathrm{OXA}-51}$ was present in 46 isolates, $b l a_{\text {OXA-23 }}$ gene was present in 44 isolates and $b l a_{\mathrm{NDM}}$ gene was found in only one strain. Other drug-resistant genes including $b l a_{\mathrm{KPC}}, b l a_{\mathrm{IMP}}$, $b l a_{\mathrm{OXA}-24}, b l a_{\mathrm{OXA}-58}, b l a_{\mathrm{SHV}}, b l a_{\mathrm{GIM}}$ and $b l a_{\mathrm{VIM}}$ were not detected in the isolates.

Of the five genes associated with the drug efflux pump tested, all five were found to be present in the isolates. Several mutations were found in the sequences of adeS gene in isolates with efflux phenotype. Differences were observed at three places when nucleotide sequences were translated into an amino acid sequence. This amino acid sequence was then
Table II. The drug-resistant rates of imipenem-resistant Acinetobacter baumannii.

\section{Resistance rate}

Drug No. of resistant strains (\%)

\begin{tabular}{ll}
\hline Meropenem & $41(89)$ \\
Amikacin & $38(83)$ \\
Piperacillin & $46(100)$ \\
Ceftazidime & $46(100)$ \\
Minocycline & $34(74)$ \\
Ciprofloxacin & $45(98)$ \\
Ampicillin/sulbactam & $43(93)$ \\
Piperacillin/tazobactam & $46(100)$ \\
Colistin & $1(2)$ \\
Trimethoprim/sulfamethoxazole & $43(93)$ \\
Cefotaxime & $45(98)$ \\
\hline
\end{tabular}

compared to the amino acid sequence of the reference strain ATCC17978 (Fig. 1). 
A

\begin{tabular}{|c|c|c|c|}
\hline Sbjet & 2936379 & 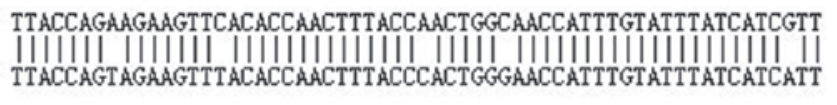 & $\begin{array}{l}60 \\
2936438\end{array}$ \\
\hline 1ery & 61 & GT & 117 \\
\hline bjet & 2936439 & GGATTTTGTTTTCTTCAGCACGTAAGTCAGCATC-CG-CATT-ACCACCTGCAGCATT & 2936495 \\
\hline 1ery & 118 & GAAACAAGCTTAACTGTTGGGTTACCAGTATAGTHAGCGCTAC & 177 \\
\hline bjet & 2936496 & AACAHAGTACCTTGTTTATCTAACTCTACAGTTGGGTACCAGTGTAGTAAGCGCCTAC & 2936555 \\
\hline very & 178 & TGG & 237 \\
\hline Sbjet & 2936556 & TTCACCALATACACCCCAGTTTTTATT GATTTTAGGIGCGAHCCARAACCTALGTACGG & 2936615 \\
\hline 1ery & 238 & & 295 \\
\hline bjet & 2936616 & TARG-Á & 2936666 \\
\hline 1ery & 296 & TTAHATC & 354 \\
\hline Sbjet & 2936667 & -TAGTTAGGTC & 2936720 \\
\hline 1ery & 355 & & 414 \\
\hline Sbjet & 2936721 & ATAKTCGTTATCTALATACGCCGCACCTGCAGCTACATATALGCCTTGAGCCAACGGTT & 2936780 \\
\hline sery & 415 & AHACGTTGTTATTATCCATATC & 474 \\
\hline Sbjet & 2936781 & TTGGCGAHTCTCAGCATTTALATATACGTTGTTATTATCCATATC & 2936840 \\
\hline Query & 475 & AATGTCACCGCCGTT & 534 \\
\hline Sbjet & 2936841 & TAATTGATAAGTCATCTCGCCAHGAAATGTCACCACCGTT & 2936900 \\
\hline Query & 535 & AGCACCACCGTAACC & 594 \\
\hline Sbjet & 293690 & AAGCACCACCGTAACC & 2936960 \\
\hline Query & 595 & GCATA & 654 \\
\hline Sbjet & 2936961 & GCACGAGCGCCTACTGGALTTAATTGGTTTTTATCGALTGCATA & 29370 \\
\hline Query & 655 & TCATCCGCCAT & \\
\hline Sbjet & 2937021 & $\mathrm{RT}$ & \\
\hline
\end{tabular}

B

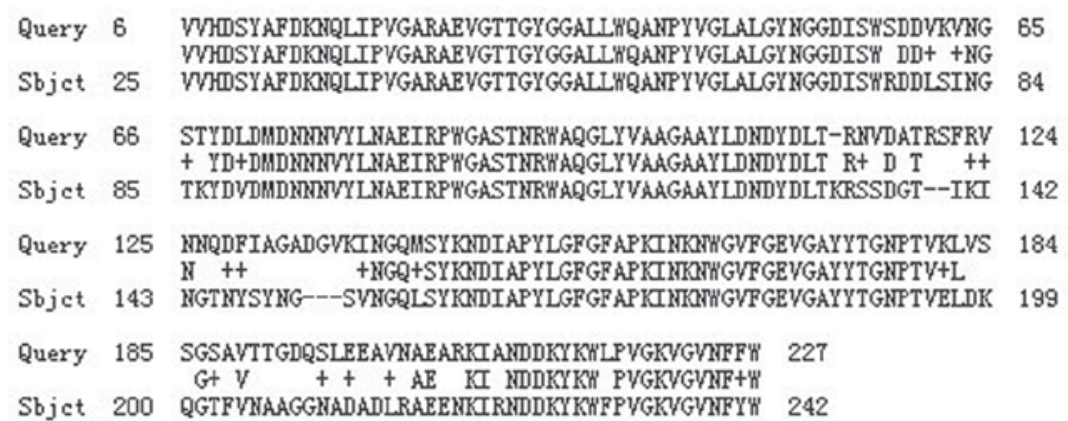

Figure 2. (A) Comparison of nucleotide sequence of $\mathrm{CarO}$ gene between resistant and reference strain ATCC17978. (B) Comparison of amino acid sequence of $\mathrm{CarO}$ gene between resistant and reference strain ATCC17978.

Similarly, the nucleotide sequence of the outer membrane protein encoding gene $\mathrm{Car} O$, when compared with the nucleotide sequence of reference strain ATCC17978, harbored mutations that were reflected in the amino acid sequence (Fig. 2).

Genotyping of isolates by MLST. The MLST analysis revealed that the isolates were clustered in 11 different genotypes or STs. The ST208 genotype was shared by the majority of isolates $(58.7 \%, 27 / 46)$, followed by ST191 $(10.9 \%, 5 / 46)$ and ST451 $(6.5 \%, 3 / 46)$. We also detected some other STs shared by certain isolates such as ST75 $(2.1 \%, 1 / 46)$, ST90 $(4.2 \%$,
2/46), ST92 (2.1\%, 1/46), ST108 (2.1\%, 31/46), ST109 (2.1\%, $1 / 46), \operatorname{ST} 172(2.1 \%, 1 / 46)$, ST368 $(4.2 \%, 2 / 46)$ and ST69 (4.2\%, $2 / 46)$. These STs were grouped into the three clonal complexes, CC92, CC109 and CC28.

\section{Discussion}

A. baumannii develops resistance to imipenem through a variety of mechanisms. Carbapenemase is an important factor responsible for imipenem resistance. In the present study, common carbapenemases were detected in the 
isolates, including $b l a_{\mathrm{OXA}-51}$, bla $_{\mathrm{OXA}-23}$, bla $_{\mathrm{OXA}-24}, b l a_{\mathrm{OXA}-58}$, $b l a_{\mathrm{KPC}}, b l a_{\mathrm{IMP}}, b l a_{\mathrm{SHV}}, b l a_{\mathrm{GIM}}, b l a_{\mathrm{NDM}}$ and $b l a_{\mathrm{VIM}}$. OXA-type enzymes are naturally present in Acinetobacter spp. and are usually expressed in small amounts (13). The expression of such genes is markedly higher under the effect of a strong promoter (insertion sequence ISAbal is the most shared) and induce drug resistance only when combined with a reduction in outer membrane permeability and/or activation of the efflux pump (14). In the present study, $b l a_{\text {OXA-51 }}$ and $b l a_{\text {OXA-23 }}$ genes were prevalent among the isolates, results that are consistent with other reports (15-17). Carbapenemases that are different from OXA, such as KPC, IMP, SHV, GIM, NDM and VIM have strong carbapenem-hydrolysing activity (14). However, such types of carbapenemases were rarely detected in $A$. baumannii. The bla $a_{\mathrm{NDM}}$ gene was identified in only one strain while the remaining resistant genes were not detected. NDM was first identified in Escherichia coli and Klebsiella pneumoniae in 2008 in India (18). This finding was followed by reports on NDM-producing P. aeruginosa, Enterobacter cloacae, Citrobacter freundii and Enterococcus faecium (19-23). In China, NDM-producing A. baumannii was first reported in 2011. Of the antimicrobials tested one NDM-positive isolate in the present study was identified that was multidrug-resistant, and only susceptible to amikacin, colistin and minocycline.

Drug efflux systems including AdeABC, AdeIJK, AdeDE and AdeXYZ (RND family) have been found in A. baumannii. Of these, the AdeABC efflux system is common in A. baumannii (24). This efflux pump, together with other resistant mechanisms, can lead to high-level imipenem resistance. Although mediated by the substrate, its expression may increase when a single point mutation occurs in the adeR or adeS gene (25). PA $\beta \mathrm{N}$ was proven to be an effective inhibitor of drug efflux. In the present study, ade $A, a d e B$ and $a d e C$ were present in all of the isolates because when PA $\beta \mathrm{N}$ was added the MICs inherent to imipenem in 13 isolates were decreased. The adeS gene differed from the adeS of standard strain and this is the possible reason for increased drug efflux associated with drug resistance.

Few studies concerning the impact of changes on membrane proteins in A. baumannii are available. In 2002, a laboratory in Argentina advocated for the first time that inducible resistance by imipenem can trigger loss of a $29-\mathrm{kDa}$ membrane protein. In 2005, the same laboratory furthering their study, demonstrated that the outer membrane protein is encoded by the $\mathrm{CarO}$ gene and when there is an insertion mutation or any other mutation in the $\mathrm{CarO}$ gene makes it off and thus the strain become resistant to certain drugs (26). In the present study, the sequence of $\mathrm{CarO}$ gene had nucleotide insertions, deletions and point mutations in comparison with the standard strains and there were also differences in their nucleotide and amino acid sequences.

In summary, for a global epidemiologic analysis, a comparison of the results between different laboratories is required. MLST is a powerful tool used to transfer typing data and compare results via relevant databases. The MLST analysis revealed that the major epidemic clone of A. baumannii in Shanghai was ST208 (CC92 clone complex), which differed from the results obtained in other regions in China (27).

\section{Acknowledgements}

The project was supported by a grant from the Natural Science Foundation of Shanghai Science and Technology Committee (no. 12ZR1426200), the Medical Guide Program of Shanghai Science and Technology Committee (no. 14411962900), Key project of Shanghai Municipal Health and Family Planning Commission (no. 201540367) and Central Universities Basic Research Program (no. 1511219024).

\section{References}

1. Perez F, Hujer AM, Hujer KM, Decker BK, Rather PN and Bonomo RA: Global challenge of multidrug-resistant Acinetobacter baumannii. Antimicrob Agents Chemother 51: 3471-3484, 2007

2. Giamarellou H, Antoniadou A and Kanellakopoulou K: Acinetobacter baumannii: A universal threat to public health? Int J Antimicrob Agents 32: 106-119, 2008.

3. Wang F, Zhu D, Hu F, Jiang X, Hu Z, Li Q, Sun Z, Chen Z, $\mathrm{Xu}$ Y, Zhang X, et al: CHINET 2012 surveillance of bacterial resistance in China. China J Infect Chemother 13: 321-330, 2013 (In Chinese).

4. Kim YJ, Kim SI, Hong KW, Kim YR, Park YJ and Kang MW: Risk factors for mortality in patients with carbapenem-resistant Acinetobacter baumannii bacteremia: Impact of appropriate antimicrobial therapy. J Korean Med Sci 27: 471-475, 2012.

5. Go ES, Urban C, Burns J, Kreiswirth B, Eisner W, Mariano N, Mosinka-Snipas K and Rahal JJ: Clinical and molecular epidemiology of acinetobacter infections sensitive only to polymyxin B and sulbactam. Lancet 344: 1329-1332, 1994

6. Pournaras S, Markogiannakis A, Ikonomidis A, Kondyli L, Bethimouti K, Maniatis AN, Legakis NJ and Tsakris A: Outbreak of multiple clones of imipenem-resistant Acinetobacter baumannii isolates expressing OXA-58 carbapenemase in an intensive care unit. J Antimicrob Chemother 57: 557-561, 2006.

7. Kuo LC, Teng LJ, Yu CJ, Ho SW and Hsueh PR: Dissemination of a clone of unusual phenotype of pandrug-resistant Acinetobacter baumannii at a university hospital in Taiwan. J Clin Microbiol 42: 1759-1763, 2004.

8. Xu J, Sun Z, Li Y and Zhou Q: Surveillance and correlation of antibiotic consumption and resistance of Acinetobacter baumannii complex in a tertiary care hospital in northeast China, 2003-2011. Int J Environ Res Public Health 10: 1462-1473, 2013.

9. Kwon NY, Kim JD and Pai HJ: The resistance mechanisms of b-lactam antimicrobials in clinical isolates of Acinetobacter baumannii. Korean J Intern Med 17: 94-99, 2002.

10. García-Quintanilla M, Pulido MR, Moreno-Martínez P, Martín-Peña R, López-Rojas R, Pachón J and McConnell MJ: Activity of host antimicrobials against multidrug-resistant Acinetobacter baumannii acquiring colistin resistance through loss of lipopolysaccharide. Antimicrob Agents Chemother 58: 2972-2975, 2014.

11. Chen TL, Siu LK, Wu RC, Shaio MF, Huang LY, Fung CP, Lee CM and Cho WL: Comparison of one-tube multiplex PCR, automated ribotyping and intergenic spacer (ITS) sequencing for rapid identification of Acinetobacter baumannii. Clin Microbiol Infect 13: 801-806, 2007.

12. Clinical Laboratory Standards Institute (CLSI): Performance standards for antimicrobial susceptibility testing; Twenty-second informational supplement. CLSI document M100-S22. CLSI, Wayne, PA, 2012.

13. Zhang JP, Zhu W, Tian SF, Chu YZ and Chen BY: Molecular characteristics and resistant mechanisms of imipenem-resistant Acinetobacter baumannii isolates in Shenyang, China. J Microbiol 48: 689-694, 2010.

14. Merkier AK and Centrón D: bla(OXA-51)-type beta-lactamase genes are ubiquitous and vary within a strain in Acinetobacter baumannii. Int J Antimicrob Agents 28: 110-113, 2006.

15. He C, Xie Y, Zhang L, Kang M, Tao C, Chen Z, Lu X, Guo L, Xiao Y, Duo L, et al: Increasing imipenem resistance and dissemination of the ISAba1-associated blaOXA-23 gene among Acinetobacter baumannii isolates in an intensive care unit. J Med Microbiol 60: 337-341, 2011. 
16. Martínez P and Mattar S: Imipenem-resistant Acinetobacter baumannii carrying the ISAba1-bla OXA-23,51 and ISAba1-bla ADC-7 genes in Monteria, Colombia. Braz J Microbiol 43: 1274-1280, 2012.

17. Corvec S, Poirel L, Naas T, Drugeon H and Nordmann P. Genetics and expression of the carbapenem-hydrolyzing oxacillinase gene blaOXA-23 in Acinetobacter baumannii. Antimicrob Agents Chemother 51: 1530-1533, 2007.

18. Yong D, Toleman MA, Giske CG, Cho HS, Sundman K, Lee K and Walsh TR: Characterization of a new metallo-beta-lactamase gene, bla(NDM-1), and a novel erythromycin esterase gene carried on a unique genetic structure in Klebsiella pneumoniae sequence type 14 from India. Antimicrob Agents Chemother 53: 5046-5054, 2009.

19. Cabanes F, Lemant J, Picot S, Simac C, Cousty J, Jalin L, Naze F, Boisson V, Cresta MP, André H, et al: Emergence of Klebsiella pneumoniae and Salmonella metallo-beta-lactamase (NDM-1) producers on reunion island. J Clin Microbiol 50: 3812, 2012.

20. Savard P, Gopinath R, Zhu W, Kitchel B, Rasheed JK, Tekle T, Roberts A, Ross T, Razeq J, Landrum BM, et al: First NDM-positive Salmonella sp. strain identified in the United States. Antimicrob Agents Chemother 55: 5957-5958, 2011.

21. Jovcic B, Lepsanovic Z, Suljagic V, Rackov G, Begovic J, Topisirovic L and Kojic M: Emergence of NDM-1 metallo- $\beta$-lactamase in Pseudomonas aeruginosa clinical isolates from Serbia. Antimicrob Agents Chemother 55: 3929-3931, 2011.

22. Poirel L, Dortet L, Bernabeu S and Nordmann P: Genetic features of blaNDM-1-positive Enterobacteriaceae. Antimicrob Agents Chemother 55: 5403-5407, 2011.
23. Ho PL, Lo WU, Yeung MK, Lin CH, Chow KH, Ang I, Tong AH, Bao JY, Lok S and Lo JY: Complete sequencing of pNDM-HK encoding NDM-1 carbapenemase from a multidrug-resistant Escherichia coli strain isolated in Hong Kong. PLoS One 6: e17989, 2011.

24. Chu YW, Chau SL and Houang ET: Presence of active efflux systems AdeABC, AdeDE and AdeXYZ in dierent AbeM (MATE) Ade ABC Acinetobacter genomic DNA groups. J Med Microbiol 55: 477-478, 2006.

25. Marchand I, Damier-Piolle L, Courvalin P and Lambert T: Expression of the RND-type efflux pump AdeABC in Acinetobacter baumannii is regulated by the AdeRS two-component system. Antimicrob Agents Chemother 48: 3298-3304, 2004.

26. Mussi MA, Limansky AS and Viale AM: Acquisition of resistance to carbapenems in multidrug-resistant clinical strains of Acinetobacter baumannii: Natural insertional inactivation of a gene encoding a member of a novel family of $\beta$-barrel outer membrane proteins. Antimicrob Agents Chemother 49: 1432-1440, 2005

27. Zhou Z, Du X, Wang L, Yang Q, Fu Y and Yu Y: Clinical carbapenem-resistant Acinetobacter baylyi strain coharboring blaSIM-1 and blaOXA-23 from China. Antimicrob Agents Chemother 55: 5347-5349, 2011. 